\title{
RHIC 28 MHZ ACCELERATING CAVITY SYSTEM*
}

\author{
J. Rose, J.M. Brennan, A. Campbell ${ }^{1}$, S. Kwiatkowski ${ }^{2}$, W. Pirkl ${ }^{3}$, A. Ratti ${ }^{2}$ \\ BNL, Upton, NY 11973, USA
}

\begin{abstract}
The $28 \mathrm{MHz}$ accelerating system consists of a quarter wave cavity driven by an inductively coupled $100 \mathrm{~kW}$ tetrode amplifer and $1 \mathrm{~kW}$ solid state driver amplifer. $40 \mathrm{~dB}$ of $\mathrm{rf}$ feedback closed around the cavity and amplifers reduces small perturbations within the loop by a factor of 100, and reduces the time required to shift the phase at transition by a factor of 10 , limited by the saturation of the drive chain. The cavity is tuned over a $200 \mathrm{kHz}$ range by a mechanical tuner which varies the gap capacitance. Broadband HOM damping is provided by two orthogonal loop coupled high pass filters. Design parameters and commissioning results are presented.
\end{abstract}

\section{INTRODUCTION}

The Relativistic Heavy Ion Collider consists of two intersecting synchrotron rings, which accelerate and collide ion species from protons to gold. The RHIC accelerating system must capture the injected beam, accelerate it through transition to top energy, and shorten the bunches prior to rebucketing into the $197 \mathrm{MHz}$ storage RF system. There are two accelerating systems per ring, each providing $300 \mathrm{kV}$ of voltage. A drawing of the cavity with its tetrode amplifier and power coupling loop and vacuum window is shown in figure 1.

\section{CAVITY}

The cavity is a capacitively loaded quarter wave structure. The capacitive loading is implemented in a coaxial geometry, which was economical to fabricate, and also has proven to be resistant to multipactor. It is constructed of copper plated carbon steel. Major cavity parameters are given in Table 1 . The cavity has interior dimensions of $0.84 \mathrm{~m}$ diameter by 2.04 meters length. The diameter was constrained by the $0.9 \mathrm{~m}$ separation of the two rings at the cavity locations. This resulted in a cavity design with a nominal shunt impedance of $\sim 1 \mathrm{M} \Omega$. The required $300 \mathrm{kV}$ can be achieved with approximately 45 $\mathrm{kW}$ of drive power. Maximum power density on the cavity wall, at the root of the inner conductor, is less than $3 \mathrm{~W} / \mathrm{cm}^{2}$. The cavity inner conductor consists of two concentric steel tubes. The inner tube has cooling channels machined on the outside, and the outer tube is then heat-shrunk over the first, and welded closed forming a water to vacuum seal on the high voltage end. This assembly is inserted into the shorting wall of the quarter wave cavity so that the water feed connections to the cooling channels are external to the assembly. Stiffening gussets are welded to stiffen the assembly. The outer cylinder, also of steel, has cooling channels welded to the outside. The coaxial capacitive loading, which is a separate spool piece, which mates to the outer cylinder, completes the major assembly. The design has proven to be quite flexible, as demonstrated when after the original design was complete the harmonic number for the accelerating system for RHIC was changed from 342 to 360 , with the cavity frequency following from $26.7 \mathrm{MHz}$ to $28.1 \mathrm{MHz}$. This was accomplished by changing only the capacitor spool piece. Provision for tuning during manufacture was made by leaving extra material on the inside bore of the coaxial spool piece, and performing final machining after assembly and frequency measurements. This precaution was not necessary as the frequency of the cavity was within $10 \mathrm{kHz}$ of the design value after machining to the predicted dimension, well within acceptable limits.

The power window consists of a $330 \mathrm{~mm}$ diameter aluminum oxide disk in a coaxial feed-through. The inner and outer coaxial vacuum seals are made with a Helicoflex Delta seal mating directly to the ceramic, without metalization. Ports were included on the cavity wall for mounting Varian Ti-ball assemblies to sublimate titanium on the window/power loop assembly in situ. This procedure was performed successfully for the first of the four cavities, which had a stubborn multipacting band. The multipacting was eliminated, even after disassembly and cleaning of the window. It is now believed that there was some contamination of this first window since none of the other cavities displayed this behavior.

Table 1: Cavity Parameters

\begin{tabular}{|l|l|}
\hline Frequency $(\mathrm{MHz})$ & 28.1 (nominal) \\
\hline Gap Voltage $(\mathrm{kV})$ & 300 \\
\hline Shunt Impedance $(\mathrm{M} \Omega)$ & $0.98(1.03$ theoretical $)$ \\
\hline Quality Factor & $15400(16162$ theoretical $)$ \\
\hline Stored Energy (joules) & 4 \\
\hline Max E-field $(\mathrm{MV} / \mathrm{m})$ & 7.8 \\
\hline Max H-field $(\mathrm{A} / \mathrm{m})$ & 6390 \\
\hline
\end{tabular}

\section{AMPLIFIERS}

The drive chain consists of a power tetrode driven by a $1 \mathrm{~kW}$ solid-state driver amplifier. A feedback loop around the cavity-amplifier chain provides $40 \mathrm{~dB}$ of RF feedback. This feedback loop reduces small perturbations due to tuner movement or beam induced transients by a factor of 100. It also reduces the time required to change the accelerating voltage phase at transition by a factor of 10 ,

\footnotetext{
*Work supported by DOE

$\dagger, 2,3$ currently at Texas Instruments, LBL, and CERN, respectively
} 
limited by the saturation of the drive chain. The cavity only requires a nominal $46 \mathrm{~kW}$ of power for $300 \mathrm{kV}$ gap volts; however during the transition phase jump the instantaneous power delivered is tripled. The tetrode amplifier is inductively coupled to the cavity. It is housed with its filament transformer and $\mathrm{RF}$ choke in an aluminum cabinet, which is jacked into position mating to the cavity coupling, loop though a coaxial connection. The amplifier uses an EIMAC 4CW150000E tetrode tube in a grounded cathode configuration. The driver amplifier is based on the two stage $1 \mathrm{~kW}$ solid-state $A B$ class amplifier described in Motorola applications note AN758 ${ }^{2}$. It consists of four output stages using Motorola MRF151G TMOS FET transistors driven by a single stage of the same configuration. This device has excellent matching characteristics and high power gain, which minimizes the number of stages, and therefore the total delay, which is an important issue in maintaining phase margin of the $40 \mathrm{~dB}$ fast-feedback. A commercial 100 $\mathrm{mW}$ pre-driver is included in the front-end. A diode limiter on the input limits the input power in case of an open loop fault, which occurs if the cavity arcs or multipactors. This was found to be insufficient to protect the driver amplifier stages from the 100fold increase of drive voltage and so each stage had its own feedback gain increased to operate near saturation at maximum power output to prevent overdriving of subsequent stages during faults.

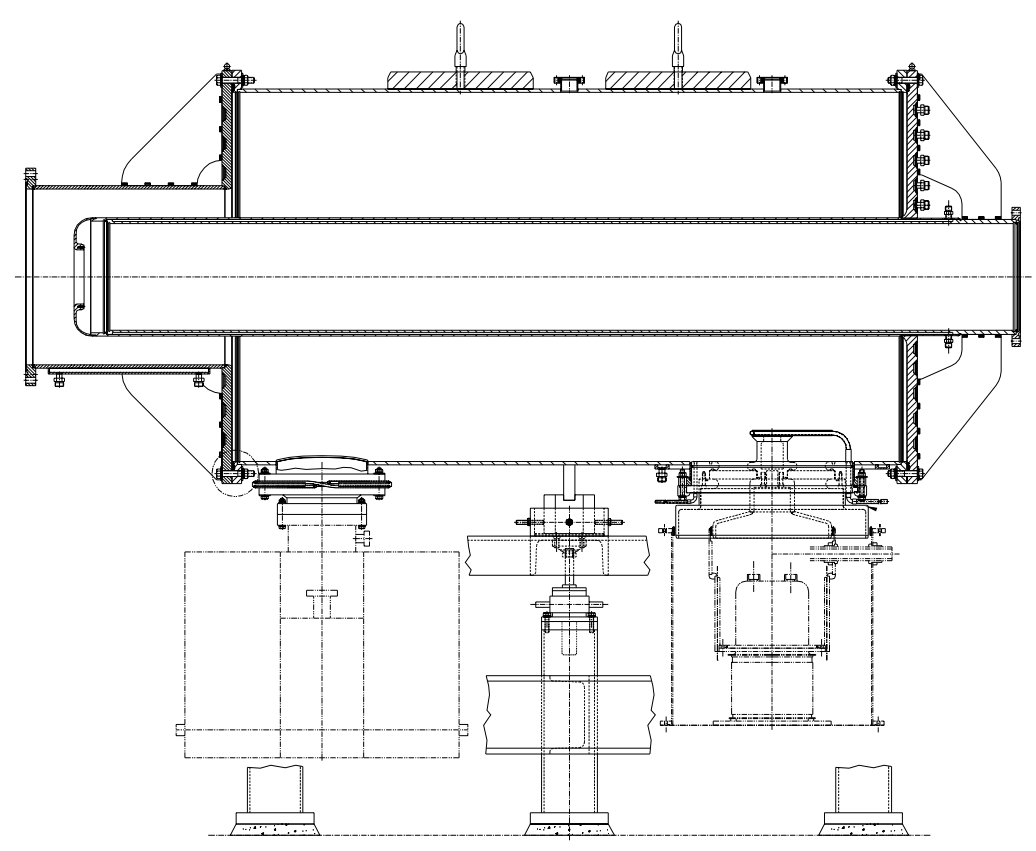

Figure 1 The RHIC $28 \mathrm{MHz}$ accelerating cavity showing vacuum pump and power tetrode. The tuner and HOM dampers are not shown in this view.

\section{TUNER}

The RF frequency in a synchrotron is given by:

$f_{r f}=\frac{h c}{C} \times \sqrt{1-\frac{1}{\gamma^{2}}}$

Where $\mathrm{h}$ is the harmonic number (360 for the accelerating system) $\mathrm{c}$ the speed of light and $\mathrm{C}$ the circumference. During the acceleration of gold ions the RF frequency increases by $90 \mathrm{kHz}$. After adding margin for thermal effects a tuning range of $230 \mathrm{kHz}$ was chosen. The relatively slow ramp of the superconducting magnets results in a maximum tuning rate of $22 \mathrm{kHz} / \mathrm{sec}$, which allowed the use of a mechanical tuner. Due to the relatively low magnetic field at the outer wall compared to the total stored energy inductive (magnetic field) tuners were impractical. It was therefore decided to tune using the high stored energy of the gap capacitance. A commercial linear stage driven by a dc servo motor drives the tuner which rides on linear bearings.

Pre-tuning of the cavity to the drive frequency is required due to the narrow bandwidth of the cavity $(\sim 1 \mathrm{kHz} 3 \mathrm{~dB}$ half-bandwidth) combined with the $40 \mathrm{~dB}$ of feedback. Otherwise the system would trip if power were applied and the cavity resonance more than a couple of bandwidths from the drive frequency. The pre-tuning was implemented by creating a lookup table of cavity frequency versus tuner position read back voltage, and then reading the drive frequency digital word from the Direct Digital Synthesizer (DDS) resident in the VME control system. The frequency is corrected for the steel cavity's temperature coefficient of $0.311 \mathrm{kHz} /$ degree $\mathrm{C}$. The program then points to the correct tuner position voltage in the lookup table.

This voltage value is inverted, then output from a VME DAC and summed with the tuner position read back to 


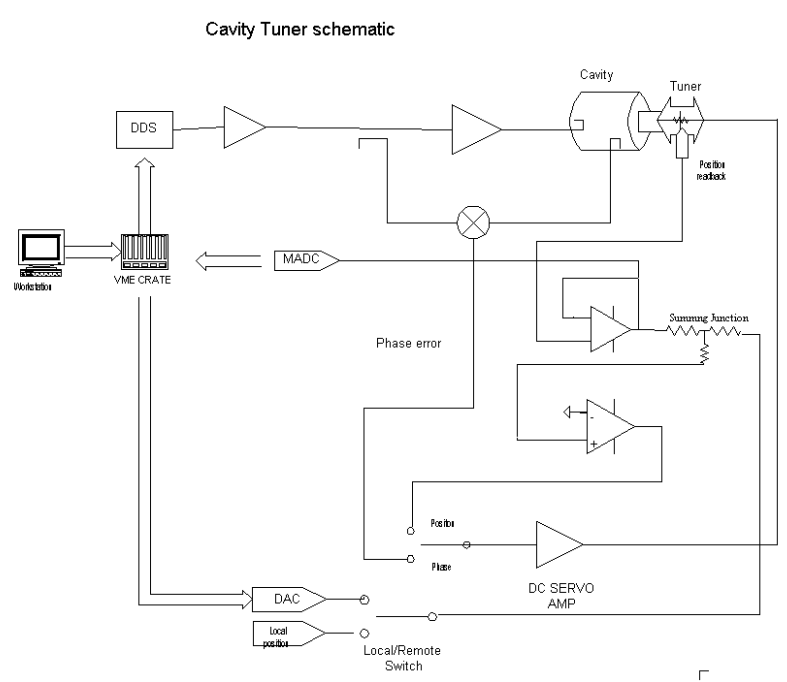

Figure 2: Block Diagram of Cavity Tuner Control

drive the tuner DC servo drive. The system automatically switches from this frequency/position loop to a phase lock loop when the cavity voltage reaches an acceptable limit.

\section{HIGHER ORDER MODE DAMPERS}

The impedance limits for higher order modes were determined and passive damping of the cavity modes was required[3]. Two concepts were prototyped. The first was a folded quarter wave notch filter to reject the fundamental. The second[4] was a more conventional high pass filter with elements lumped elements constructed within a $6 \quad 1 / 8$ inch coaxial line. Both prototypes performed acceptably. The high pass approach chosen because its low $\mathrm{Q}$ nature meant less sensitivity to mis-tuning for the rejection of the fundamental, and a perhaps mistaken view that it would not dissipate as much power. Loop-coupled high pass filters were designed, and two installed orthogonal to each other to effectively damp both monopole and dipole cavity modes. The loops are installed through the enwall of the cavity and extend up through the cylindrical wall where they couple to a $5^{\text {th }}$ order $\sim$ Chebyschev high pass filter ${ }^{3}$, with a cut-off frequency of $67 \mathrm{MHz}$. The schematic is shown in figure 3.

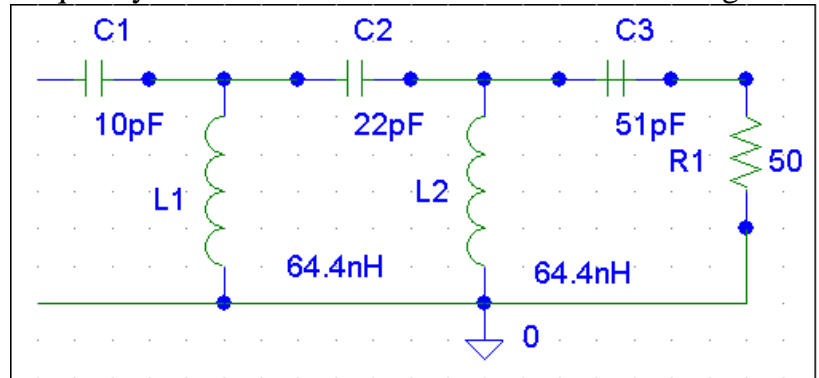

Figure 3. PSPICE Schematic of HOM High-pass Filter.

The first capacitor, which is in series with the loop inductance, is reduced to below that which would cause voltage breakdown in air. The filter was realized in $61 / 8$ inch coaxial transmission line. The first element is a parallel plate capacitor composed of two disks, the bottom of which is mounted to a conflat flange which blanks off the cylindrical vacuum window feed through. The cross section of the filter is shown in Fig.2. The second and third capacitors are coaxial, the inner conductor being the same as for the $61 / 8$ inch coaxial line, and the outer cylinders machined of brass. Rexolite was used as the dielectric material. Spiral arms between the inner and outer conductors formed the inductances. Since the output end of the filter is in EIA 6 1/8-inch coax a standard taper to N-type connector and a commercial 10-Watt load terminates the filter. Fundamental mode is rejected in the filter by more than $60 \mathrm{~dB}$. The maximum fundamental frequency voltage at the coupling loop of HOM damper is $7.3 \mathrm{kV}$ (for a gap voltage of $300 \mathrm{kV}$ ).

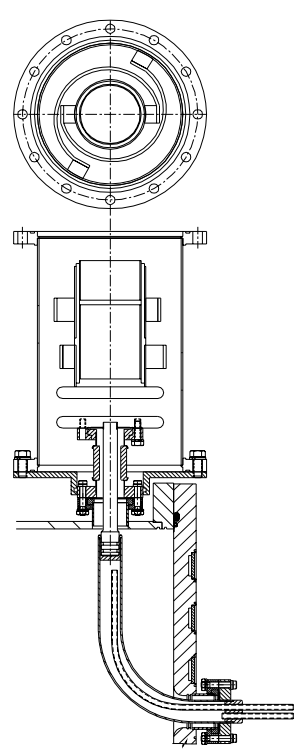

Figure 4. Drawing of HOM showing loop coupling, vacuum feed through and high-pass filter.

\section{Acknowledgements}

The authors would like to acknowledge the technical excellence of the RHIC RF group in carrying out the above work and that of Pierre Maccioni and the staff at CERCA in the manufacture of the cavities.

\section{REFERENCES}

[1] J. Rose, J. Browdowski, D.P. Deng, S. Kwiatkowski, W. Pirkl, A. Ratti "Design of the $26.7 \mathrm{MHz}$ RF Cavity for RHIC" Particle Accelerator Conference, 1995 Dallas, TX

[2] H. Granberg "A Two-Stage $1 \mathrm{~kW}$ Solid State Linear Amplifier" Motorola Applications Note AN-758

[3] J. Rose "Calculations of HOM's and Coupled Bunch Instabilities due to the RHIC RF Cavities" EPAC 94

[4] S. Kwiatkowski, J. Rose "HOM Damper for RHIC 26.7 MHz Accelerating System" RHIC/RF Technical Note No. 25 